\title{
Local rhamnosoft, ceramides and L-isoleucine in atopic eczema: a randomized, placebo controlled trial
}

\author{
Alessia Marseglia ${ }^{1}$, Amelia Licari ${ }^{1}$, Fabio Agostinis ${ }^{2}$, Antonio Barcella ${ }^{3}$, Domenico Bonamonte ${ }^{4}$, \\ Mario Puviani ${ }^{5}$, Massimo Milani ${ }^{6}{ }^{2}$ GianLuigi Marseglia ${ }^{1}$ \\ ${ }^{1}$ Policlinico San Matteo, Pediatric Clinic, Pavia, Italy; ${ }^{2}$ Ambulatorio di Dermatologia, Pediatric Clinic, Bergamo, Italy; ${ }^{3}$ Ambulatorio di \\ Dermatologia, Nembro, Bergamo, Italy; ${ }^{4}$ Department of Biomedical Science and Human Oncology - Section of Dermatology, University of Bari \\ "Aldo Moro", Bari, Italy; ${ }^{5}$ Ospedale Di Sassuolo, Struttura Semplice Di Dermatologia E Dipartimento Di Dermatologia Chirurgica, Sassuolo, Italy; \\ ${ }^{6}$ Isdin Medical Department Milan, Milan, Italy
}

To cite this article: Marseglia A, Licari A, Agostinis F, Barcella A, Bonamonte D, Puviani M, Milani M, Marseglia G. Local rhamnosoft, ceramides and L-isoleucine in atopic eczema: a randomized, placebo controlled trial. Pediatr Allergy Immunol 2014: 14: 271-275.

\section{Keywords}

atopic eczema; emollient therapy;

randomized trial; assessor-blinded

\section{Correspondence}

Massimo Milani, Medical Department Isdin Italy, Viale Abruzzi 3, 20100 Milan

Tel.: +390220520276

Fax: +390229411286

E-mail: massimo.milani@isdin.com

Accepted for publication 3 December 2013

DOI:10.1111/pai.12185

\begin{abstract}
Background: A non-steroidal, anti-inflammatory moisturizing cream containing rhamnosoft, ceramides, and L-isoleucine (ILE) (pro-AMP cream) has been recently developed for the specific treatment of atopic eczema (AE) of the face. In this trial, we evaluated the clinical efficacy and tolerability of pro-AMP cream in the treatment of facial AE in children in comparison with an emollient cream.

Methods: In a randomized, prospective, assessor-blinded, parallel groups (2:1) controlled trial, 107 children (72 allocated to pro-AMP cream and 35 allocated to control group) with mild-to-moderate chronic AE of the face were enrolled. Treatments were applied twice daily for a 6-week period. Facial Eczema Severity Score (ESS) was evaluated at baseline, week 3, and week 6, by an assessor unaware of treatment allocation. Investigator's Global Assessment (IGA) score was assessed at week 3 and at week 6 . Tolerability was evaluated at week 3 and at week 6 using a 4-point score (from 0: low tolerability to 3: very good tolerability).

Results: At baseline ESS, mean (SD) was 6.1 (2.4) in the pro-AMP cream group and 5.3 (3) in the control group. In the pro-AMP group, in comparison with baseline, ESS was significantly reduced to $2.5(-59 \%)$ after 3 wks and to $1.0(-84 \%)$ at week 6 $(\mathrm{p}=0.0001)$. In the control group, ESS was reduced to $3(-42 \%)$ at week 2 and to 2.6 $(-50 \%)$ at week 6 . At week 6, ESS in pro-AMP cream was significantly lower than the control group (1.0 vs. $2.6 ; \mathrm{p}=0.001)$. Both products were well tolerated.

Conclusion: Pro-AMP cream has shown to be effective in the treatment of mild-tomoderate chronic lesion of $\mathrm{AE}$ of the face. Clinical efficacy was greater in comparison with an emollient cream. (Clinical trial Registry: NTR4084).
\end{abstract}

Atopic eczema (AE) is a very common disease in pediatric population (1). AE is a chronic inflammatory itchy skin condition that develops in the majority of cases in the first year of life (2). It is typically an episodic disease of exacerbation (flares, which may occur as frequently as two or three per month) and remissions, except for severe cases where it may be continuous (3). Skin barrier alteration (4) and reduction in innate immune mechanisms (5) (low production of antimicrobial peptides: AMP) are considered the hallmarks of AE. Bacterial colonization, favored by reduction in innate immune mechanisms and skin barrier alteration, is linked with severity and exacerbation of AE (6). The face is frequently affected in AE representing a therapeutic challenge (7). Face AE limits the use, especially for long periods, of corticosteroid topical products for the high risk of atrophic skin changes (8). A non-steroidal, antiinflammatory moisturizing cream containing rhamnosoft, ceramides, and L-isoleucine (ILE) has been recently developed for the specific treatment of AE of the face. Rhamnosoft is a rhamnose-rich polysaccharide with high affinity to keratinocyte receptors sharing anti-inflammatory and antibacterial adhesion properties (9). Topical ceramides could restore the altered skin barrier in AE (10). Topical ILE has shown to stimulate a skin level production of AMP $(11,12)$. In addition, ILE is also able to potentiate the functional activities of $\beta$-defensin, increasing its chemoattractant activity (13). Therefore, this topical formulation has a strong rational for the use in $\mathrm{AD}$ subjects. In addition, this composition 
could exert a stronger clinical effect in comparison with simple emollient or hydrating topical products used in this clinical setting.

\section{Study aim}

We evaluated the clinical efficacy and tolerability of a $2.5 \%$ rhamnosoft (Biosaccharide GUM-2), ceramides (ceramide 3), and $2 \%$ ILE containing cream (Nutratopic Pro-AMP cream, Isdin Barcelona, Spain) in the treatment of facial atopic eczema in children in comparison with a simple hydrating cream $(15 \%$ glycerol-based cream containing also vaseline $8 \%$ and liquid paraffin 2\%) (Clinical Trial registry: NTR4084).

\section{Methods}

In a no-profit, multicenter, randomized, prospective, assessorblinded, parallel groups (with imbalanced treatment allocation 2:1) controlled trial, 107 children between the age of 6 months to $14 \mathrm{yr}$ with mild-to-moderate chronic AE of the face were enrolled (72 allocated to pro-AMP cream and 35 allocated to control group). Diagnosis of AE was performed according to Hanifin and Rajka criteria (14). Main exclusion criteria were severe $\mathrm{AE}$, recent (i.e., $<4$ wks) treatment with systemic or topical steroids or calcineurin inhibitors, and the presence of active cutaneous bacterial, viral, or fungal infections in target areas. Computer-generated number randomization list was used for treatment allocation. Study took place in $5 \mathrm{~s}$ and third-level outpatient dermatology pediatric services in Italy between January and May 2013. The two topical treatments were applied on the face twice daily (two fingertip units per application) for a 6 -week period. Specific instructions how to apply topical study products were given to subjects/parents at the baseline visit. Outcomes of the study were the Eczema severity score, Investigator Global assessment and the tolerability score. Facial Eczema Severity Score (ESS) was evaluated by an assessor (a physician with particular expertise in AD) unaware of treatment allocation at baseline, week 3, and week 6. The intensity of redness (erythema), thickness (induration, papulation, edema), scratching (excoriation), and lichenification (lined skin) of the eczema was assessed and scored as none or absent $(0)$, mild (1), moderate (2), and severe (3) according to Hanifin et al. (15). Half scores were not allowed. Investigator's Global Assessment (IGA) (16) score was assessed at week 3 and at week 6 . Tolerability was evaluated at week 3 and at week 6 using a 4-point score. Protocol-specified primary outcome of the study was the evolution of facial 4-point ESS (from $0=$ no sign, to 3 = severe). Secondary outcomes were the Investigator Global assessment score, graded on a scale of $0-4 \quad(0=$ clear, $1=$ almost clear, $2=$ mild disease, 3 = moderate disease, $4=$ severe) evolution, and the treatment success percentage in the two groups at the end of study treatment, defined as an IGA score of 0 or 1 . Parent/tutor informed consent was obtained for each patient before entering in the trial. Study protocol was approved by local IRB.

\section{Statistical analysis and sample size calculation}

Statistical analysis was performed using Graphpad ${ }^{\mathrm{TM}}$ (San Diego, CA, USA) statistical software. We used Mann-Whitney test for the primary outcome evaluation (evolution of ESS) and for the IGA score. The Fisher's exact test was used for the comparison of success treatment percentage in the two groups. Continuous data are expressed as means and standard deviations (SDs). The study was designed as a superiority trial. Study hypothesis was that mean ES score, the primary outcome of the trial, at week 6 would be significantly lower in pro-AMP cream treated group (experimental group) in comparison with control group. If the true difference in the experimental and control means would be at least -0.45 (effect size), we needed to study at least 60 experimental subjects and 30 control subjects to be able to reject the null hypothesis that the population means of the experimental and control groups are equal with probability level (power) of 0.9 . The type I error probability associated with this test of the null hypothesis is 0.5. Sample size calculation was performed using PS Power and Sample Size ${ }^{\circledR}$ Calculations Version 3.0 software.

\section{Results}

All 107 enrolled patients (59 boy and 48 girls; mean age 6 yr; range 6 months-2 yr) concluded the 6-week study period. Table 1 summarizes subjects' characteristics at baseline. Seventy patients were allocated to pro-AMP group and 35 to emollient cream control group. History of AE lasted $6 \mathrm{yr}$ in the pro-AMP group and $5 \mathrm{yr}$ in the control group. The two groups were well balanced regarding the main demographic and medical variables. At baseline ES score, mean (SD) was 6.1 (2.5) in children in the pro-AMP cream group and 5.3 (3) in the control group. In the pro-AMP cream treated group, in comparison with baseline, facial ESS was significantly reduced to 2.5 (2) (representing a $59 \%$ reduction) after $3 \mathrm{wks}$ and to 1.0 (1.5) (representing a $-84 \%$ reduction) at week $6(\mathrm{p}=0.0001)$ with an absolute reduction of -5.0 points $(95 \% \mathrm{CI}$ : from -5.5 to -4.5 ). In the control group, ESS was reduced to 3 (2) (representing a $-42 \%$ reduction) at week 3 and to 2.6 (2)

Table 1 Subjects' characteristics at baseline

\begin{tabular}{lcc}
\hline & $\begin{array}{c}\text { Pro-AMP } \\
\text { group }\end{array}$ & $\begin{array}{c}\text { Control } \\
\text { cream } \\
\text { group }\end{array}$ \\
\hline $\mathrm{N}$ & 72 & 35 \\
Male/female & $36 / 36$ & $23 / 12$ \\
Age, years, mean (SD) & $6(6)$ & $5.8(5)$ \\
Eczema severity score, & $6.1(2-12)$ & $5.3(2-12)$ \\
$\quad$ mean (range) & $6(5)$ & $5(6)$ \\
History of atopic eczema, & & \\
$\quad$ years, mean (SD) & & \\
History of respiratory allergies (rhinitis/asthma) & 11 \\
$\quad$ Yes & 25 & 25 \\
$\quad$ No & 47 & 100 \\
Attending to school or kindergarten, \% & 100 & \\
\hline
\end{tabular}


$(-50 \%)$ at week 6 with an absolute reduction of -2.7 points (95\% CI: from -3.6 to -1.4$)$. At week 6 , facial ESS in proAMP cream was significantly lower than the control group (1.0 vs. 2.6; $\mathrm{p}=0.0001$ ) (Fig. 1a). IGA score was 1.5 and 0.6 at week 3 and 6 , respectively, in pro-AMP cream group. In control group, IGA score was 1.6 at week 3 and 1.2 at week 6 . IGA score at week 6 was lower in pro-AMP cream group in comparison with control group (0.6 vs. $1.2 ; \mathrm{p}=0.0003)$ (Fig. 1b). Treatment success (IGA score $\leq 1$ ) percentage at week 6 was $88 \%$ in pro-AMP group and $58 \%$ in the control group ( $\mathrm{p}=0.009$; Fisher's exact test) with a number needed to treat of 3.3 in favor of pro-AMP group. Both products were well tolerated with a tolerability score of 2.8 in pro-amp cream group and 2.9 in the control group (NS).

\section{Discussion}

The epidermal barrier is an important component of the defensive function of the skin (17). The main skin protective functions are related to water loss modulation and prevention,

(a) Evolution of facial Eczema severity score

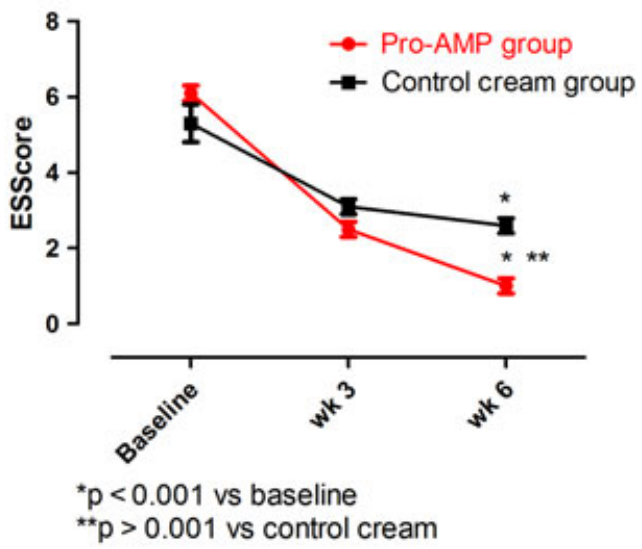

(b)

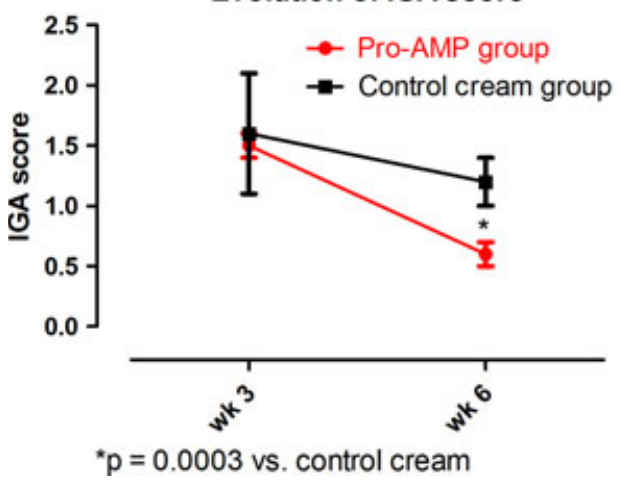

Figure 1 (a) Evolution of Eczema severity Score from baseline to week 3 and week 6 in the two study groups. Data presented as mean \pm SEM. $* p<0.001$ vs. baseline, $* * p>0.001$ vs. control cream. (b) Evolution of Investigator Global Assessment (IGA) score at week 3 and week 6 in the two study groups. Data presented as mean \pm SEM. $* p=0.0003$ vs. control cream.
UV-protection, anti-oxidant, and antimicrobial actions (18). Epidermal barrier function is abnormal in subjects suffering from atopic dermatitis (19). Skin barrier defect in AE is mainly due to a reduced lipid (i.e., ceramide) content of epidermis (20), a reduced or genetically altered filaggrin synthesis (21) and finally to a reduced synthesis of AMP (5). Skin barrier function alteration correlates with AE severity (22). For these reasons, treatments with the aim to improve skin barrier properties of AE subjects could be a relevant approach in the strategic treatment of this skin condition (23). In view of the fact that $\mathrm{AE}$ is a chronic condition, caring for atopic skin must be an everyday task. An efficient skin care may reduce acute flares by improving the compromised skin barrier and reducing TEWL (Trans Epidermal Water Loss) (24). Moisturizers have been shown to improve TEWL through restoration of the integrity of the stratum corneum, acting as a barrier to water loss and replacement of skin lipids and other compounds (25). For these reasons, emollient and hydrating products are considered a cornerstone treatment of AE to improve skin barrier function (26). Guidelines recommend the regular use of emollients and skin protectants for the prevention and maintenance of the epidermal skin barrier in patients with AE (27); emollients may even reduce the need for topical corticosteroid use (28). A 3-month open trial (29) using daily emollient treatment has shown to improve both clinical and quality of life in mild AE patients. Patrizi et al. (30) in a vehicle-controlled 3-arm clinical trial performed in a total of 60 children with AE have shown that emollient treatment induced an $80 \%$ improvement in the IGA score in comparison with baseline. In a more recent trial, Hon et al. (31) have shown that 2 -week ceramide-containing emollient product treatment induced a $21 \%$ reduction in SCORAD in 24 patients with AE. However, this clinical effect in patients with Staphylococcus aureus colonization was lower in comparison with patients without bacterial colonization. The use of topical anti-inflammatory and moisturizing products containing also compounds, which could improve the innate immunologic system of the skin such as isoleucine, could be a further step in the rational topical treatment approach in AE (32). In this study, we have evaluated the clinical efficacy of a non-steroidal, anti-inflammatory, moisturizing cream containing rhamnosoft, ceramides, and Lisoleucine in the treatment of mild-to-moderate $\mathrm{AE}$ of the face. In comparison with simple emollient/hydrating topical products, this formulation, from a theoretic point of view, could exert in AE patients multiple mechanisms of action such as a skin barrier protective effect, an anti-inflammatory action, and a potentiation of innate immune system of the skin. Facial ESS score after 6-week treatment with this product decreased by $80 \%$ in comparison with baseline with a greater efficacy in comparison with simple emollient cream. Some limitations should be taken into account in evaluating the results of the present study. First, this was not a double-blinded study. To perform a double-blinded study, taking into account the formulation and texture differences between the topical products used, a double dummy procedure should be adopted. We overcome this problem using an assessor-blinded approach in assessing primary and secondary outcomes. Treatment duration was $6 \mathrm{wks}$, and therefore, no data regarding efficacy and 
tolerability for longer treatment durations could be inferred from this study. However, similar trials available in the literature performing in this clinical setting have evaluated very often shorter treatment periods (i.e., $<4$ wks) $(33,34)$ or were carried out in a limited number of subjects (35). One strength point of the present study is the sample size, which is so far one of the largest performed in comparative evaluation of the efficacy of emollient treatments in AE pediatric patients. Hon et al. (24) in a systematic review of controlled clinical studies with barrier repair therapies in AE underline the fact that these trials generally suffered from a lack of sample size calculation and small sample size. In addition, in these studies, treatment effects were generally small or marginal. In our trial, sample size was calculated according to the clinical hypothesis to be tested (ES score difference vs. control emollient treatment). Therefore, on evaluating the results of our study, the risk of a Type II error could be considered quite low. In addition, taking into account inclusion and exclusion criteria used in our study and the clinical setting in which the trial was carried out, the results we have obtained could be considered to have a good external validity level. Our study demonstrated that a non-steroidal cream containing rhamnosoft, ceramides, and L-isoleucine has shown to be effective in the treatment of mild-to-moderate chronic lesion of $\mathrm{AE}$ of the face. Clinical efficacy was greater in comparison with a simple emollient/ hydrating cream.

\section{Acknowledgments}

This was a non-profit study. MM is an employee of Isdin Srl. He was involved in the study design and in the manuscript preparation. Isdin $\mathrm{Srl}$ has kindly provided the products used in the trial. The other authors have declared no conflict of interest.

\section{Author Contributions}

Conceived and designed the study: GLM, DB, and MM. Performed the trial: AM, MP, AB, FA, DB. Wrote the paper: GLM, MM.

\section{References}

1. Shen CY, Lin MC, Lin HK, Lin CH, Fu LS, $\mathrm{Fu}$ YC. The natural course of eczema from birth to age 7 years and the association with asthma and allergic rhinitis: a populationbased birth cohort study. Allergy Asthma Proc 2013: 34: 78-83.

2. Burr ML, Dunstan FD, Hand S, Ingram JR, Jones KP. The natural history of eczema from birth to adult life: a cohort study. $\mathrm{Br}$ J Dermatol 2013: 168: 1339-42.

3. Darsow U, Wollenberg A, Simon D, et al. ETFAD, EADV eczema task force 2009 position paper on diagnosis and treatment of atopic dermatitis. J Eur Acad Dermatol Venereol 2010: 24: 317-28.

4. Elias PM, Hatano Y, Williams ML. Basis for the barrier abnormality in atopic dermatitis: outside-inside-outside pathogenic mechanisms. $J$ Allergy Clin Immunol 2008: 121: 1337-43.

5. Ong PY, Ohtake T, Brandt C, et al. Endogenous antimicrobial peptides and skin infections in atopic dermatitis. $N$ Engl $J$ Med 2002: 347: 1151-60.

6. Jinnestål CL, Belfrage E, Bäck O, Schmidtchen A, Sonesson A. Skin barrier impairment correlates with cutaneous Staphylococcus aureus colonization and sensitization to skin-associated microbial antigens in adult patients with atopic dermatitis. Int J Dermatol 2014: 53: 27-33.

7. Bieber T. Atopic dermatitis. $N$ Engl J Med 2008: 358: 1483-94.

8. Lee NP, Arriola ER. Topical corticosteroids: back to basics. West J Med 1999: 171: 351-3.

9. Andrès E, Molinari J, Péterszegi G, et al. Pharmacological properties of rhamnose-rich polysaccharides, potential interest in agedependent alterations of connectives tissues. Pathol Biol (Paris) 2006: 54: 420-5.

10. Jungersted JM, Agner T. Eczema and ceramides: an update. Contact Dermatitis 2013: 69: 65-71.

11. Sherman H, Chapnik N, Froy O. Albumin and amino acids upregulate the expression of human beta-defensin 1. Mol Immunol 2006: 43: 1617-23.

12. Fehlbaum P, Rao M, Zasloff M, Anderson GM. An essential amino acid induces epithelial beta-defensin expression. Proc Natl Acad Sci U S A 2000: 97: 12723-8.

13. Tyrrell C, De Cecco M, Reynolds NL, et al. Isoleucine/leucine 2 is essential for chemoattractant activity of beta-defensin Defb 14 through chemokine receptor 6. $\mathrm{Mol}$ Immunol 2010: 47: 1378-82.

14. Hanifin JM, Rajka G. Diagnostic features of atopic dermatitis. Acta Derm Venereol (Stockh) 1980: 92: 44-7.

15. Hanifin JM, Thurston M, Omoto M, Cherill R, Tofte SJ, Graeber M. The eczema area and severity index (EASI): assessment of reliability in atopic dermatitis. EASI Evaluator Group. Exp Dermatol 2001: 10: 11-8.

16. Gelmetti C, Colonna C. The value of SCORAD and beyond. Towards a standardized evaluation of severity? Allergy 2004: 59(Suppl. 78): 61-5.

17. Elias PM, Feingold KR. Lipids and the epidermal water barrier: metabolism, regulation, and pathophysiology. Semin Dermatol 1992: 11: 176-82.

18. Proksch E, Brandner JM, Jensen JM. The skin: an indispensable barrier. Exp Dermatol 2008: 17: 1063-72.
19. Elias PM, Schmuth M. Abnormal skin barrier in the etiopathogenesis of atopic dermatitis. Curr Opin Allergy Clin Immunol 2009: 9: 437-46.

20. Imokawa G, Abe A. decreased level of ceramides in stratum corneum of atopic dermatitis: an etiologic factor in atopic dry skin? J Invest Dermatol 1991: 96: 523-6.

21. O'Regan GM, Sandilands A, McLean WH, Irvine AD. Filaggrin in atopic dermatitis. J Allergy Clin Immunol 2008: 122: 689-93.

22. Gupta J, Grube E, Ericksen MB, et al. Intrinsically defective skin barrier function in children with atopic dermatitis correlates with disease severity. $J$ Allergy Clin Immunol 2008: 121: 725-30.

23. Elias PM, Feingold KR. Does the tail wag the dog? Role of the barrier in the pathogenesis of inflammatory dermatoses and therapeutic implications. Arch Dermatol 2001: 137: 1079-81.

24. Hon KL, Leung AK, Barankin B. barrier repair therapy in atopic dermatitis: an overview. Am J Clin Dermatol 2013: 14: 389-99.

25. Nolan K, Marmur E. Moisturizers: reality and the skin benefits. Dermatol Ther 2012: 25: 229-33.

26. Varothai S, Nitayavardhana S, Kulthanan K. Moisturizers for patients with atopic dermatitis. Asian Pac J Allergy Immunol 2013: 31: 91-8.

27. Rubel D, Thirumoorthy T. Consensus guidelines for the management of atopic dermatitis: an Asia-Pacific perspective. $J$ Dermatol 2013: 40: 160-71. 
28. Grimalt R, Mengeaud V, Cambazard F. The steroid-sparing effect of an emollient therapy in infants with atopic dermatitis: a randomized controlled study. Dermatology 2007: 214: 61-7.

29. Gelmetti C, Boralevi F, Seité S, et al. Quality of life of parents living with a child suffering from atopic dermatitis before and after a 3-month treatment with an emollient. Pediatr Dermatol 2012: 29. 714-8.

30. Patrizi A, Capitanio B, Neri I, et al. A double-blind, randomized, vehiclecontrolled clinical study to evaluate the efficacy and safety of MAS063DP

(ATOPICLAIR) in the management of atopic dermatitis in paediatric patients. Pediatr Allergy Immunol 2008: 19: 619-25.

31. Hon KL, Pong NH, Wang SS, Lee VW, Luk NM, Leung TF. Acceptability and efficacy of an emollient containing ceramideprecursor lipids and moisturizing factors for atopic dermatitis in pediatric patients. Drugs R D 2013: 13: 37-42.

32. Elias PM, Wakefield JS. Therapeutic implications of a barrier-based pathogenesis of atopic dermatitis. Clin Rev Allergy Immunol 2011: 41: 282-95.

33. Simpson E, Böhling A, Bielfeldt S, Bosc C, Kerrouche N. Improvement of skin barrier function in atopic dermatitis patients with a new moisturizer containing a ceramide precursor. J Dermatolog Treat 2013: 24: 122-5.

34. Draelos ZD. A clinical evaluation of the comparable efficacy of hyaluronic acidbased foam and ceramide-containing emulsion cream in the treatment of mild-tomoderate atopic dermatitis. $J$ Cosmet Dermatol 2011: 10: 185-8.

35. Miller DW, Koch SB, Yentzer BA, et al. An over-the-counter moisturizer is as clinically effective as, and more costeffective than, prescription barrier creams in the treatment of children with mild-tomoderate atopic dermatitis: a randomized, controlled trial. J Drugs Dermatol 2011: 10: 531-7. 